\title{
Real-time observation of nonlinear coherent phonon dynamics in single-walled carbon nanotubes
}

\author{
A. GAMBETTA ${ }^{1}$, C. MANZONI ${ }^{1}$, E. MENNA², M. MENEGHETTI2 G. GERULLO ${ }^{1}$, G. LANZANI ${ }^{1 *}$, S. TRETIAK ${ }^{3}$, \\ A. PIRYATINSKI ${ }^{3}$, A. SAXENA ${ }^{3}$, R. L. MARTIN ${ }^{3}$ AND A. R. BISHOP ${ }^{3}$ \\ ${ }^{1}$ CNR-INFM, National Laboratory for Ultrafast and Ultraintense Optical Science, Dipartimento di Fisica, Politecnico di Milano, P.za L. da Vinci 32,20133 Milan, Italy \\ ${ }^{2}$ Department of Chemical Sciences, University of Padova, 1, Via Marzolo, 35131 Padova, Italy \\ ${ }^{3}$ Theoretical Division and Center for Nonlinear Studies, Los Alamos National Laboratory, Los Alamos, New Mexico 87545, USA \\ *e-mail: guglielmo.lanzani@fisi.polimi.it
}

$(0)$ ingle-walled carbon nanotubes (SWNTs) are $\pi$-conjugated, quasi-one-dimensional structures consisting of rolled-up graphene sheets that, depending on their chirality, behave as semiconductors or metals ${ }^{1}$; owing to their unique properties, they enable groundbreaking applications in mechanics, nanoelectronics and photonics, ${ }^{2,3}$. In semiconducting SWNTs, medium-sized excitons $(3-5 \mathrm{~nm})$ with large binding energy and oscillator strength are the fundamental excitations ${ }^{4-8}$; exciton wavefunction localization and one-dimensionality give rise to a strong electron-phonon coupling ${ }^{9-11}$, the study of which is crucial for the understanding of their electronic and optical properties. Here we report on the use of resonant sub-10-fs visible pulses ${ }^{12}$ to generate and detect, in the time domain, coherent phonons in SWNT ensembles. We observe vibrational wavepackets for the radial breathing mode (RBM) and the G mode, and in particular their anharmonic coupling, resulting in a frequency modulation of the G mode by the RBM. Quantumchemical modelling ${ }^{13}$ shows that this effect is due to a corrugation of the SWNT surface on photoexcitation, leading to a coupling between longitudinal and radial vibrations.

Electron-phonon coupling in SWNTs is usually studied using Raman spectroscopy; this technique is useful for investigating ground-state vibrations ${ }^{14}$, whereas photoexcited-state vibrational dynamics remain largely unknown because, in the frequency domain, phonon replicas are hardly detectable in the presence of substantial inhomogeneous broadening. Time-domain observation of phonon dynamics has much lower sensitivity with respect to conventional Raman, but it enables direct measurement of excitedstate dynamics, vibrational dephasing and mode coupling in a distinct way ${ }^{15,16}$. Coherent phonon detection allows resolution in time of wavepacket dynamics that is otherwise averaged-out in standard Raman scattering.

To detect coherent phonons in SWNTs, we use a standard pump-probe configuration, in which the observed quantity is the modulation depth in the differential transmission ${ }^{17}(\Delta T / T)$; details of the experimental setup are provided in the Methods section. Figure la shows $\Delta T / T$ dynamics of SWNTs grown by the high-pressure carbon monoxide procedure dispersed in polymethylmethacrylate films following excitation with a sub-10-fs visible pulse (1.8-2.4 eV bandwidth), probed at an energy of $2.1 \mathrm{eV}$. The signal exhibits an initial photobleaching, which quickly turns into photoinduced absorption (PA). The fast photobleaching decay is ascribed to relaxation of the higher-lying exciton (second in an increasing energy scale) to the lower one, taking place with a $40-\mathrm{fs}$ time constant ${ }^{18}$. The PA signal is generated by this lower exciton ${ }^{4,5}$ and decays on the ps timescale, in agreement with previous results ${ }^{19-21}$. As shown in Fig. 1a, there is a clear oscillation in the $\Delta T / T$ amplitude. The Fourier transform (FT) of the oscillatory component (Fig. 2a) shows a strong peak at $252 \mathrm{~cm}^{-1}(132-\mathrm{fs}$ period). This frequency can be recognized as the RBM, associated with expansion and contraction of the tube cross-section ${ }^{14}$. The observation is related to an ensemble of semiconducting SWNTs, with diameters of about $0.95 \mathrm{~nm}$, all vibrating in phase. A similar result is obtained pumping with a 30 -fs pulse in the near-infrared $(0.92 \mathrm{eV})$ range and probing in the visible $(2 \mathrm{eV})$ range with a sub-10-fs pulse (Fig. 1b). Here, the lowest exciton is directly excited and its PA rises promptly; a clear modulation at the frequency of $259 \mathrm{~cm}^{-1}$ is visible on top of it.

The 'displacive excitation' mechanism ${ }^{22}$ can account for coherent phonon generation in SWNTs: carbon atoms do not have time to move during the short pulse excitation. However, in the excited-state electronic configuration, they may have a different equilibrium position. This will occur only for those modes that do not distort the molecular symmetry during vibration, namely totally symmetric modes (those observed in optical transitions and resonance Raman scattering). Calculations based on a semiempirical excited-state molecular dynamics (ESMD) approach ${ }^{13}$ confirm that both the RBM and $G$ modes have substantial coupling with the electronic transitions. Calculated dimensionless displacements from the ground state to the lowest optically active 


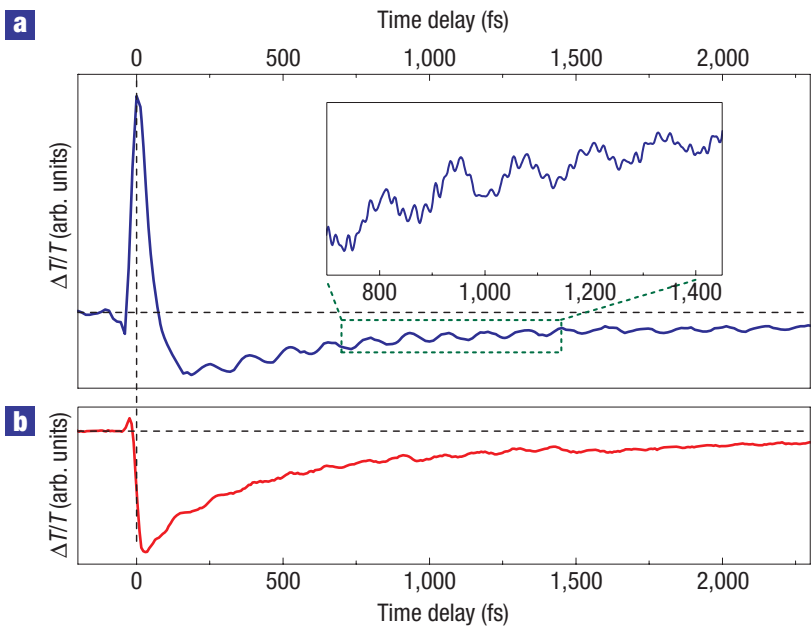

Figure 1 Differential transmission $(\Delta T / T)$ dynamics of SWNTs. a, Excitation and probing by a sub-10-fs visible pulse; probe energy is $2.1 \mathrm{eV}$. The inset shows a portion of the trace acquired with a higher sampling rate, to fully resolve the high-frequency modulation. b, Excitation by a $30-\mathrm{fs}$ pulse at $0.92 \mathrm{eV}$ and probing by a sub-10-fs visible pulse; probe energy is $2.0 \mathrm{eV}$.

exciton state are 0.2 for the RBM and 0.4 for the G mode. As a consequence, the atoms oscillate along the selected configurational coordinate minimum. Unless coherence is destroyed by intraand/or inter-tube perturbations, the vibrational motion of the ensemble is that of a single-molecule trajectory. All of the nanotubes involved breathe in phase, by periodically modulating their diameter. We find that the semiconducting nanotubes which can be excited in our experiments are $(10,3),(9,5),(9,4)$ and $(7,6)$ with RBM frequencies 253,242, 257 and $263 \mathrm{~cm}^{-1}$ in the ground state and the second exciton resonance at 1.95, 1.85, 2.03 and $1.92 \mathrm{eV}$, respectively ${ }^{23}$. This justifies the observation of oscillations with frequencies around $250 \mathrm{~cm}^{-1}$. The lack of RBM modes at $200 \mathrm{~cm}^{-1}$ indicates that coherence is not generated in metallic tubes, although they are present in our sample. The modulation depth decays slowly, with time constant $T_{2} \approx 1.6 \mathrm{ps}$, allowing more than 20 oscillations to be detected. Analogous to nanoelectromechanical systems ${ }^{24}$, this phenomenon suggests that SWNTs can be light-driven nanoscale oscillators, behaving as molecular springs at $7.5 \mathrm{THz}$ frequency, actuated by an optical pulse. Our results demonstrate that coherent control of structural modifications in SWNTs is indeed feasible ${ }^{25}$.

The inset of Fig. 1a shows a portion of the $\Delta T / T$ trace acquired with a higher sampling rate (2.66-fs steps) and indicates that there is also modulation at high frequency, corresponding, in the FT spectrum, to $1,588 \mathrm{~cm}^{-1}$ (21-fs period). This value agrees with that of the $\mathrm{G}$ band assigned to the totally symmetric longitudinal carbon-stretching mode, as shown in the comparison with the Raman spectrum (Fig. 2b).

A further tool available for analysing our data is the sliding window $\mathrm{FT}^{26}$ (SWFT), which yields instantaneous frequencies and amplitudes of the molecular vibrations. In Fig. 3a the SWFT corresponding to the trace in the inset of Fig. 1a is reported as a contour plot, clearly showing that there is a modulation in the G-mode frequency. The modulation period can be extracted by carrying out a FT of the time-dependent band peak position. The result of such an operation, shown in Fig. 3b, is striking: the modulation frequency coincides with that of the RBM. A straightforward interpretation can be given: the breathing SWNT

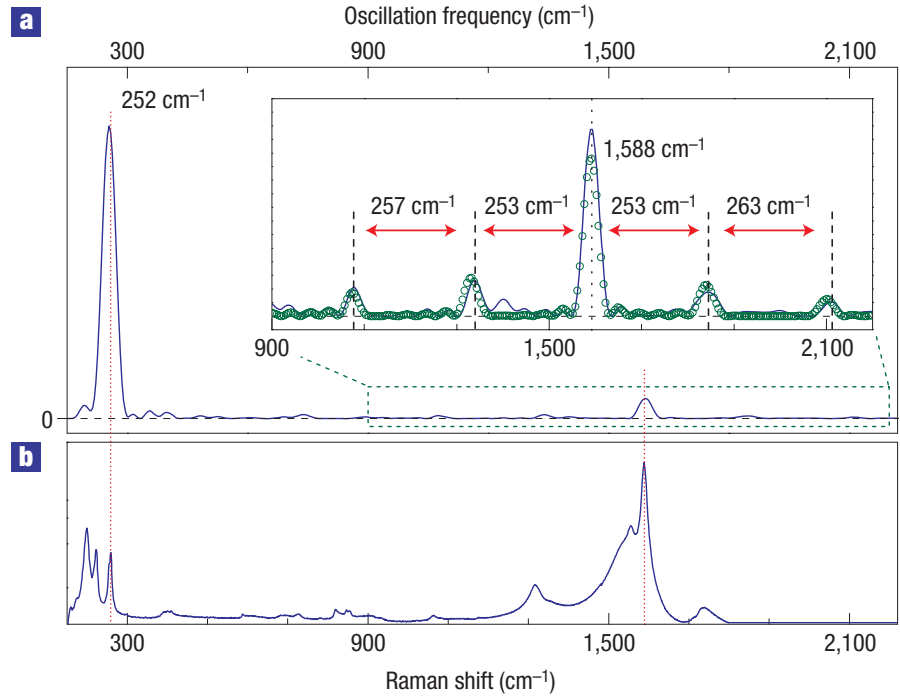

Figure 2 Vibrational spectra of SWNTs. a, FT power spectrum of time trace shown in the inset of Fig. 1a. Inset: the solid line is the zoom of the FT power spectrum, showing sidebands around the $\mathrm{G}$ mode. Regular spacing between the modes is indicated by labels and double-headed arrows. The circles are a fit according to the model described in the text. b. Continuous-wave Raman spectrum with He-Ne excitation.

sample passes through different geometries, corresponding to different potential energy curvatures for the $\mathrm{G}$ mode. The large period difference between the two modes $\left(\tau_{\mathrm{RBM}} / \tau_{\mathrm{G}}=130 / 21 \approx 6\right)$ leads to decoupling of the oscillations in the time domain so that the high-frequency mode follows the low-frequency mode adiabatically. Artefacts resulting from an overlap between the broadened FT peaks in the SWFT spectra can be disregarded because of such dynamical separation ${ }^{17}$. Frequency modulation should have a corresponding signature in the frequency domain, where sidebands are expected; accordingly, the experimental FT spectrum in the whole time range (solid line in Fig. 2a, inset), contains small peaks that do not correspond to any feature in the Raman spectrum and are almost equally spaced in wavenumbers, at $1,078,1,335,1,841$ and $2,104 \mathrm{~cm}^{-1}$, their difference being approximately that of the RBM. Note that an amplitude modulation would also lead to sidebands in the frequency domain, however with a different phase relation; thus, the critical check for distinguishing the two is the time-domain SWFT. The sidebands do not appear as in Raman spectra, where only some combination modes can be observed ${ }^{27}$. However, such bands are extremely small, and they would be subjected to ultrafast phase loss, so they could not be due to G and RBM mode coupling, that would in any case occur in the ground electronic state. As a consequence those would not give rise to any signal in the time domain. On the contrary, our experimental results suggest that the G-mode frequency depends on the RBM configurational coordinate, $\omega_{\mathrm{G}}=\omega_{\mathrm{G}}\left(Q_{\mathrm{RBM}}(t)\right)$, where $t$ is time. This dependence can easily be modelled by assuming $Q_{\mathrm{RBM}}(t)=Q_{0} \cos \left(\omega_{\mathrm{RBM}} t\right)$ and expanding to the second order, obtaining:

$$
\omega_{\mathrm{G}}(t)=\omega_{\mathrm{G} 0}\left[1+\gamma_{1} \cos \left(\omega_{\mathrm{RBM}} t\right)+\gamma_{2} \cos \left(2 \omega_{\mathrm{RBM}} t\right)\right] .
$$

Here $\omega_{\mathrm{G} 0}$ is the unperturbed G-mode frequency, and $\gamma_{1}$ and $\gamma_{2}$ are the expansion coefficients representing coupling constants with the fundamental and second harmonic of the RBM mode. 

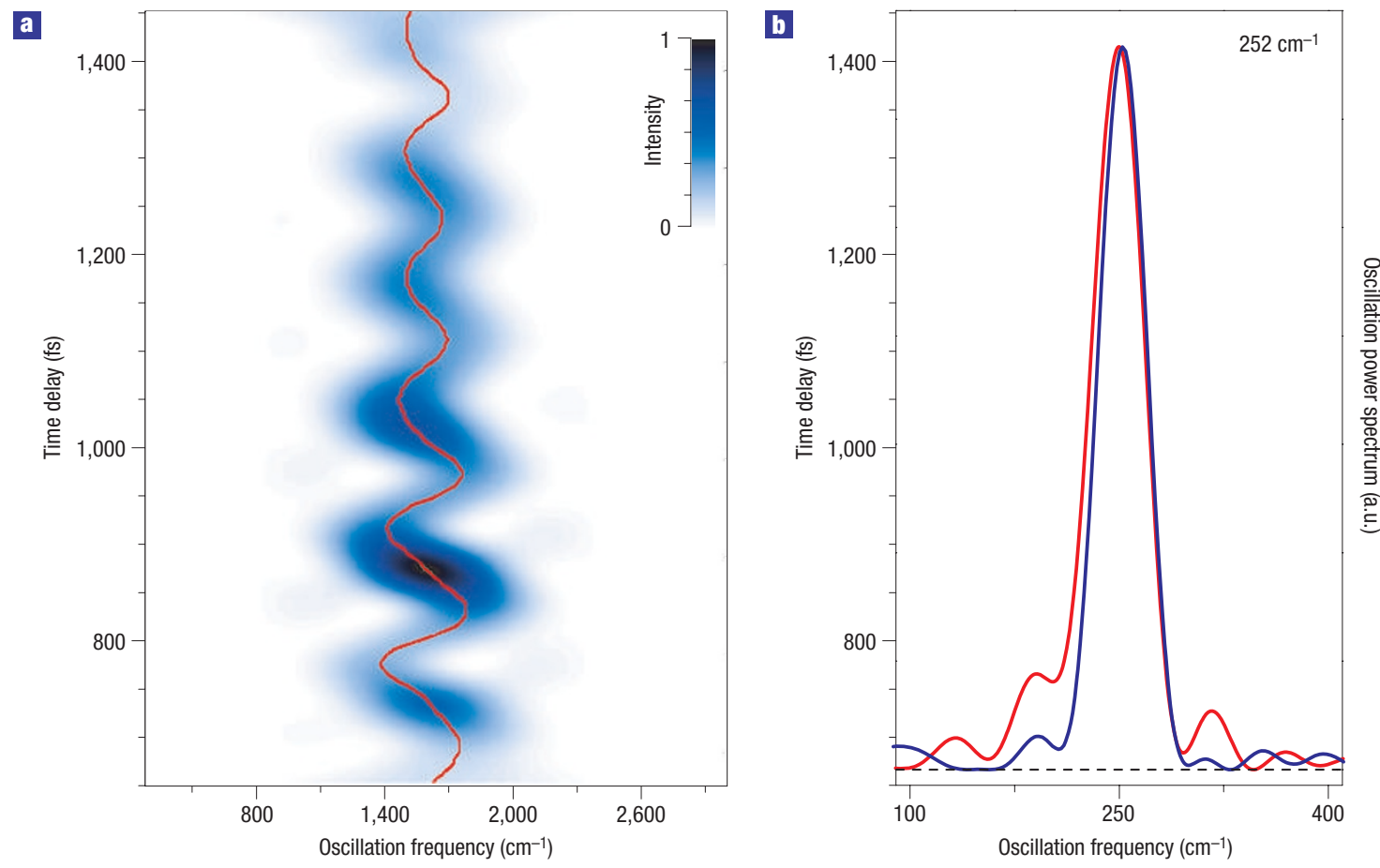

Figure 3 Analysis of the frequency modulation of the G mode. a, SWFT of the data in the inset of Fig. 1a. The measured G-mode peak position is plotted as a solid line. b, Red line: FT power spectrum of the time dependence of the band peak position from a; blue line: FT power spectrum of the time trace shown in Fig. 1a. The peak at $200 \mathrm{~cm}^{-1}$ is assigned to semiconducting nanotubes, possibly $(14,1),(15,2),(12,5)$ and $(11,7)$, which can be excited only with the wings of the visible pulse.

Using $\gamma_{1}=0.156$ and $\gamma_{2}=0.094$ we can reproduce both the FT spectrum (circles in Fig. 2a, inset) and the spectrogram. Note that a qualitative justification of the above assumption can also be derived by the two-coupled classic oscillator model (see the Methods section).

To gain insight into the effective nonlinear phonon coupling mechanism, we carried out modelling based on the ESMD approach. All reported computational results are for the chiral $(7,6)$ tube with finite boundaries, but sufficiently long $(5 \mathrm{~nm})$ to mimic the infinite tube limit and exclude edge effects from the tube ends. The simulated photoexcited trajectories have been propagated along all nuclear degrees of freedom for up to 1 ps. These calculations assume the Born-Oppenheimer approximation: nuclei are classical and electrons are quantum. No approximations to the vibrational couplings and anharmonicities were made because the underlying hamiltonian (Austin Model 1, AM1, ref. 28) has these effects incorporated in the dependence of its matrix elements on the nuclei positions. The RBM and G mode are both Raman-active Frank-Condon vibrations, that is, their equilibrium positions change on electronic excitation. However, tube-diameter modification in going from the ground to the first exciton state is not simple. In Fig. 4 a, we show the change in tube radius $d r$ with atom coordinate along tube $Z$ axis calculated for the $(7,6)$ tube shown in the inset. Overall, relaxation of the photoexcitation results in an increase in the average diameter in the middle of the tube, causing it to bulge. This reflects an exciton self-trapping phenomenon similar to that observed in conjugated polymers ${ }^{13}$. Surprisingly, there is also a corrugation of the tube surface in the excited state, which clearly leads, in support of our conjecture, to coupling of radial and longitudinal modes.

We also calculated ground state normal modes for the $(7,6)$ tube and obtained 323 and $1,737 \mathrm{~cm}^{-1}$ as RBM and G-mode frequencies, respectively. These are overestimated compared with the experiment, which is typical for AM1. From the experiment, it is possible to estimate the anharmonic coupling strength between the RBM and $G$ mode in the excited state to be about $2 \mathrm{~cm}^{-1}$. However, calculated ground-state cubic anharmonicities do not exceed $0.05 \mathrm{~cm}^{-1}$ and quartic anharmonicities do not exceed $0.01 \mathrm{~cm}^{-1}$; that is, radial and longitudinal modes are essentially uncoupled in the ground state. Direct calculation of vibrational normal modes and anharmonicities in the excited state is not possible due to the computational expense. Using ground-state normal modes as a basis, we numerically estimated frequencies of the RBM and G mode in the excited state to be 324 and $1,727 \mathrm{~cm}^{-1}$, respectively. Because of the corrugation of the tube surface in the excited state, these modes weakly mix (Duschinsky rotation), which we estimate, from the off-diagonal elements of the hessian matrix, to be about $1 \%$. Finally, to explore the anharmonic coupling between RBM and G mode in the excited state, we ran molecular dynamics simulations. The trajectories were started from ground- (excited-) state equilibrium geometries with the $\mathrm{G}$ mode excited by two vibrational quanta $(\sim 0.43 \mathrm{eV})$. In the ground state, the trajectories (not shown) are perfectly harmonic with all vibrational energy preserved in the $G$ mode on the ps timescale. The situation is very different in the excited state. Figure $4 \mathrm{~b}$ shows the FT of a 1 ps trajectory of the RBM dimensionless displacement. In addition to the fundamental RBM frequency, we observe a weak component of the excited state G-mode frequency appearing due to linear mixing of the $G$ and RBM in the excited state. Furthermore, the G-mode component in the FT spectrum (inset of Fig. 4b) contains equally spaced small satellites that correspond to respective overtones of the RBM mode, strikingly similar to Fig. 2a. This provides computational confirmation of anharmonic coupling between radial and longitudinal modes induced in the excited state. 


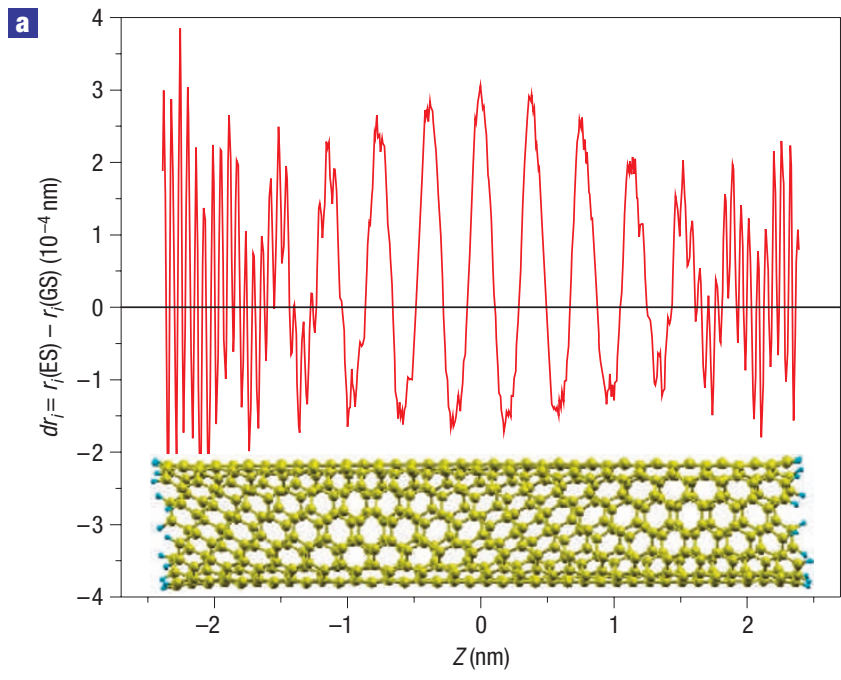

b

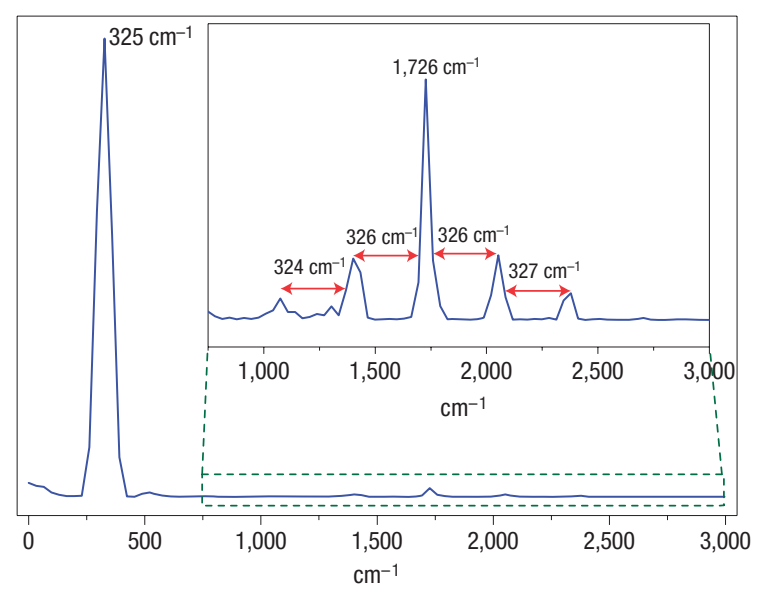

Figure 4 Quantum chemical modelling. a, Comparison of calculated excited- and ground-state optimal geometries. Modification of radius $d r$ with carbon coordinate along the tube $Z$ axis for the chiral $(7,6)$ SWNT shown in the inset. $\mathbf{b}$, FT power spectrum of the trajectory of RBM dimensionless displacement calculated using the ESMD approach. Inset: zoom-out of the FT power spectrum, showing sidebands in the high-frequency region.

In summary, this study shows that ultrashort light pulses can drive coherent atomic motion in SWNTs, in spite of the large inhomogeneous broadening. We have demonstrated the high selectivity of the mechanism: metallic tubes are not involved; essentially only two modes in semiconducting tubes are excited. With such a technique, nonlinear molecular dynamics can be observed. The exciton state modulates the phonon field introducing the nonlinearity, which manifests itself as vibrational coupling. Quantum-chemical dynamic computations show that on excitation, the nanotube's surface becomes locally distorted, inducing a coupling between the radial and longitudinal modes, which gives rise to the frequency modulation experimentally observed in the time domain.

\section{METHODS}

\section{ULTRAFAST SPECTROSCOPY}

The ultrafast spectroscopy setup is based on a non-collinear optical parametric amplifier (NOPA), pumped by an amplified Ti:sapphire laser (500 $\mu \mathrm{J}, 150 \mathrm{fs}$,
$1 \mathrm{kHz}, 780 \mathrm{~nm}$ ). In the NOPA, a fraction of 780-nm light is focused on a thin sapphire plate to generate a white-light continuum, whereas the remainder is frequency doubled to $390 \mathrm{~nm}$; parametric amplification of the white light takes place in a $\beta$-barium borate crystal, pumped by the $390-\mathrm{nm}$ beam, using a suitable non-collinear geometry that guarantees a broad gain bandwidth. The NOPA generates ultrabroadband visible pulses, with a spectrum spanning the $1.8-2.4 \mathrm{eV}$ frequency range. These pulses are then compressed to a nearly transform-limited 6-7 fs duration by multiple reflections onto specially designed chirped dielectric mirrors. The trace in Fig. $1 \mathrm{~b}$ is obtained by generating the pump pulses with a second NOPA similar to that described above, but tuned to the near-infrared $(0.92 \mathrm{eV})$ region.

Pump-probe experiments are carried out in a standard non-collinear configuration using only reflective optics. Time-resolved measurements at specific wavelengths are obtained by spectrally filtering, with 10-nm bandwidth interference filters, the probe beam after passing through the sample and combining differential detection with lock-in amplification. All measurements are carried out in air at room temperature.

\section{SAMPLE PREPARATION}

We studied samples of SWNTs grown by the high-pressure carbon monoxide procedure. After purification, buckytubes (Carbon Nanotechnologies) were functionalized with poly(ethylene glycol) chains to improve dispersion in a polymer matrix. The derivatization was achieved through amidation of nanotube-bound carboxylic acids with poly(ethylene glycol)-amine $\mathrm{e}^{29}$. The functionalized SWNTs were embedded in polymethylmethacrylate films that were cast on glass cover slips. Resonance Raman spectra showed that the oxidation reaction used for the functionalization destroyed the nanotubes with diameters below $\approx 0.85 \mathrm{~nm}$ (ref. 30 ).

\section{DATAANALYSIS}

The FTs are carried out on the oscillatory component of the signal, after subtracting a slowly varying background. The spectrograms are obtained by carrying out a SWFT, that is, a series of FTs carried out on different time windows with position swept along the whole measurement domain. The SWFT is obtained according to the formula

$$
S(\omega, \tau)=\int_{0}^{\infty} s(t) g(t-\tau) \exp (-j \omega t) \mathrm{d} t
$$

where $s(t)$ is the oscillatory component of the signal and $g(t-\tau)=\exp \left[-(t-\tau)^{n} / \tau_{\mathrm{P}}^{n}\right]$ is a super-gaussian window function. In our calculation we used $n=6$ and $\tau_{\mathrm{p}}=70 \mathrm{fs}$.

\section{DYNAMICS OF COUPLED CLASSICAL OSCILLATORS}

To illustrate the model used for experimental fitting (equation (1)) based on the parametric dependence of the G-mode frequency on the RBM configurational coordinate, we consider a very simple model of the dynamics of two coupled anharmonic classical oscillators. The general form of the system lagrangian in the normal coordinate representation $q_{i}$ is

$$
L=\sum_{i=1,2} \frac{m_{i} \dot{q}_{i}^{2}}{2}-\sum_{i=1,2} \frac{k_{i} q_{i}^{2}}{2}-\sum_{i, j, n=1,2} \frac{k_{i j n} q_{i} q_{j} q_{n}}{3 !}-\sum_{i, j, n, m=1,2} \frac{k_{i j n m} q_{i} q_{j} q_{n} q_{m}}{4 !} .
$$

Here, terms higher than quartic anharmonicities $k_{i j n m}$ have been truncated. By denoting $q_{1}=q$ as the coordinate of the G mode with mass $m_{1}=m$, spring constant $k_{1}=k$ and bare frequency $\sqrt{k / m}=\omega_{\mathrm{GB}}$, and $q_{2}=Q$ being the coordinate of the RBM with mass $m_{2}=M$, spring constant $k_{2}=K$ and frequency $\sqrt{K / M}=\omega_{\mathrm{RBM}}$ and applying the Lagrange equation, we obtain nonlinear equations of motion for the two oscillators. We can write the following equations considering, in particular, the lower-order terms which allow a description of the interaction between the two oscillators:

$$
\begin{aligned}
& \ddot{Q}+\omega_{\mathrm{RBM}}^{2} Q+\alpha Q q+\sigma Q q^{2}=0 \\
& \ddot{q}+\omega_{\mathrm{GB}}^{2} q+\beta q Q+\eta q \mathrm{Q}^{2}=0,
\end{aligned}
$$

where $\alpha=2 k_{122} / M$ and $\beta=2 k_{112} / m, \sigma=2 k_{1122} / m$, and $\eta=2 k_{1122} / M$.

According to the model we are proposing, where the $Q$ mode is a slowly varying potential for the higher frequency $q$ mode, we can assume 
$Q(t) \sim \cos \left(\omega_{\mathrm{RBM}} t\right)$. Therefore, for the $q$ mode we can write:

$$
\ddot{q}+\omega_{\mathrm{GB}}^{2}\left(1+\frac{\beta}{\omega_{\mathrm{GB}}^{2}} \cos \left(\omega_{\mathrm{RBM}} t\right)+\frac{\eta}{\omega_{\mathrm{GB}}^{2}} \cos ^{2}\left(\omega_{\mathrm{RBM}} t\right)\right) q=0 .
$$

An expansion of the oscillator frequency in powers of $\beta$ and $\eta$ up to the linear terms,

$$
\omega_{\mathrm{G}}=\omega_{\mathrm{GB}}\left(1+\frac{\beta}{\omega_{\mathrm{GB}}^{2}} \cos \left(\omega_{\mathrm{RBM}} t\right)+\frac{\eta}{\omega_{\mathrm{GB}}^{2}} \cos ^{2}\left(\omega_{\mathrm{RBM}} t\right)\right)^{1 / 2},
$$

gives the following expression for modulated G-mode frequency in the form of equation (1):

$$
\omega_{\mathrm{G}}(t)=\omega_{\mathrm{G} 0}\left(1+\gamma_{1} \cos \left(\omega_{\mathrm{RBM}} t\right)+\gamma_{2} \cos ^{2}\left(\omega_{\mathrm{RBM}} t\right)\right),
$$

with the renormalized fundamental frequency

$$
\omega_{\mathrm{G} 0}=\omega_{\mathrm{GB}}\left(1+\frac{\eta}{4 \omega_{\mathrm{GB}}^{2}}\right),
$$

and the modulation parameters

$$
\begin{gathered}
\gamma_{1}=\frac{\beta}{2 \omega_{\mathrm{GB}^{2}}}\left(1-\frac{\eta}{4 \omega_{\mathrm{GB}}^{2}}\right)\left(1+\frac{\eta}{4 \omega_{\mathrm{GB}}^{2}}\right)^{-1} \\
\gamma_{2}=\frac{\eta}{4 \omega_{\mathrm{GB}}^{2}}\left(1+\frac{\eta}{4 \omega_{\mathrm{GB}}^{2}}\right)^{-1} .
\end{gathered}
$$

Equation (2) clearly show that the value of the modulation parameters $\gamma_{1}$ and $\gamma_{2}$ is a result of the interplay between the values of the cubic and quartic anharmonicities entering through $\beta$ and $\eta$. Accordingly, their small but close values obtained in the paper from the fit of the pump-probe experiment and also confirmed by the numerical simulations, clearly indicate that both anharmonic terms are equally responsible for the mode coupling.

\section{SIGNATURES OF THE EXCITED-STATE MODE COUPLING IN THE PUMP-PROBE RESPONSE}

The pump-probe signal induced by the ultrafast non-overlapping pulses can be analysed using the doorway-window representation, which for a vibrationally coupled two-level electronic system reads ${ }^{31}$

$$
S_{p p}\left(\omega_{1} \omega_{2} ; t\right)=\sum_{e e^{\prime}} \rho_{e e^{\prime}}^{W}\left(\omega_{2}\right) G_{e e^{\prime}}(t) \rho_{e e^{\prime}}^{D}\left(\omega_{1}\right)+\sum_{g g^{\prime}} \rho_{g g^{\prime}}^{W}\left(\omega_{2}\right) G_{g g^{\prime}}(t) \rho_{g g^{\prime}}^{D}\left(\omega_{1}\right) .
$$

Each component in equation (3) is a product of three terms. First, the doorway function $\rho_{e e^{\prime}}^{D}\left(\omega_{1}\right)\left[\rho_{g g^{\prime}}^{D}\left(\omega_{1}\right)\right]$ describing the excitation of the vibrational coherence $|e\rangle\left\langle e^{\prime}\right|\left[|g\rangle\left\langle g^{\prime}\right|\right]$ in the excited [ground] electronic state by the pump pulse. Its dependence on $\omega_{1}$ mimics the optical absorption lineshape at experimental temperature. Second, $G_{e e^{\prime}}(t)\left[G_{g g^{\prime}}(t)\right]$ is the Green function describing time evolution of the vibrational coherence $|e\rangle\left\langle e^{\prime}\right|\left[|g\rangle\left\langle g^{\prime}\right|\right]$ during the delay time $t$ between the pump and probe pulses. Finally,

\section{$\rho_{e e^{\prime}}^{W}\left(\omega_{2}\right)\left[\rho_{g g^{\prime}}^{W}\left(\omega_{2}\right)\right]$ is the window function having the shape of the optical} absorption band at infinite temperature, and describing the effect of the probe pulse absorption. If the pump (probe) pulse bandwidth is narrower than the absorption bandwidths it should be convoluted with the doorway (window) functions.

The first term in equation (3) depends on $G_{e e^{\prime}}(t)$, and, therefore, carries spectroscopic signatures of the vibrational dynamics in the excited electronic state. The second one, containing $G_{g g^{\prime}}(t)$, represents the ground-state vibrational dynamics, and carries similar information on the mode coupling as Raman spectra. As a result, in the signal analysis we should focus on the first term. To identify the features observed in Fig. 1 and in the inset of Fig. 2a, we follow the assumption that the RBM and G-mode dynamics are classical. Then, the corresponding Green function is

$$
G_{\mathrm{G}}(t)=\exp \left[i \int_{0}^{t} \omega_{\mathrm{G}}(\tau) \mathrm{d} \tau-t / T_{2}\right],
$$

where $\omega_{\mathrm{G}}(t)=\omega_{\mathrm{G} 0}\left[1+\gamma_{1} \cos \left(\omega_{\mathrm{RBM}} t\right)+\gamma_{2} \cos \left(2 \omega_{\mathrm{RBM}} t\right)\right]$ is the G-mode frequency adiabatically modulated by the RBM mode, and $T_{2}$ is the dephasing time. The integral can be easily evaluated giving

$$
G_{\mathrm{G}}(t)=\exp \left[i \omega_{\mathrm{G} 0} t-t / T_{2}+\frac{i \gamma_{1} \omega_{\mathrm{G} 0}}{\omega_{\mathrm{RBM}}} \sin \left(\omega_{\mathrm{RBM}} t\right)+\frac{i \gamma_{2} \omega_{\mathrm{G} 0}}{2 \omega_{\mathrm{RBM}}} \sin \left(2 \omega_{\mathrm{RBM}} t\right)\right] .
$$

Further expanding the exponential in powers of $\gamma_{1}$ and $\gamma_{2}$, and retaining terms up to the linear one, the Green function can be recast as

$G_{\mathrm{G}}(t) \approx\left(1+\frac{i \gamma_{1} \omega_{\mathrm{G} 0}}{\omega_{\mathrm{RBM}}} \sin \left(\omega_{\mathrm{RBM}} t\right)+\frac{i \gamma_{2} \omega_{\mathrm{G} 0}}{2 \omega_{\mathrm{RBM}}} \sin \left(2 \omega_{\mathrm{RBM}} t\right)\right) \exp \left[i \omega_{\mathrm{G} 0} t-t / T_{2}\right]$.

This expression can be used to analyse the observed time-domain signal in Fig. 1.

To gain insight into the features present in the inset of Fig. 2a, the FT of equation (4) on the interval of $t=(0, \infty)$ has to be made, transforming the Green function to the following frequency-domain representation

$$
G_{\mathrm{G}}(\omega)=G_{\mathrm{G} 0}(\omega)+G_{\mathrm{G} 1}(\omega)+G_{\mathrm{G} 2}(\omega),
$$

where the terms in the sum are resonant at $\omega_{\mathrm{G} 0}, \omega_{\mathrm{G} 0} \pm \omega_{\mathrm{RBM}}$, and $\omega_{\mathrm{G} 0} \pm 2 \omega_{\mathrm{RBM}}$ respectively. By introducing the homogeneous line width $\delta=2 \pi / T_{2}$, below, we provide expressions for these terms. The main peak at $\omega=\omega_{\mathrm{G} 0}$ is described by

$$
G_{\mathrm{G} 0}(\omega)=\frac{-i}{\omega-\omega_{\mathrm{G} 0}-i \delta} .
$$

The first sidebands $\omega=\omega_{\mathrm{G} 0} \pm \omega_{\mathrm{RBM}}$ appear in

$$
G_{\mathrm{G} 1}(\omega)=\frac{i \gamma_{1} \omega_{\mathrm{G} 0}}{2 \omega_{\mathrm{RBM}}}\left(\frac{1}{\omega-\omega_{\mathrm{G} 0}+\omega_{\mathrm{RBM}}-i \delta}-\frac{1}{\omega-\omega_{\mathrm{G} 0}-\omega_{\mathrm{RBM}}-i \delta}\right),
$$

and finally, the second sidebands $\omega=\omega_{\mathrm{G} 0} \pm 2 \omega_{\mathrm{RBM}}$ are given by

$$
G_{\mathrm{G} 2}(\omega)=\frac{i \gamma_{2} \omega_{\mathrm{G} 0}}{4 \omega_{\mathrm{RBM}}}\left(\frac{1}{\omega-\omega_{\mathrm{G} 0}+2 \omega_{\mathrm{RBM}}-i \delta}-\frac{1}{\omega-\omega_{\mathrm{G} 0}-2 \omega_{\mathrm{RBM}}-i \delta}\right) .
$$

An important observation in equations (5)-(8) regarding the ratio of the peaks' absolute values can be made. Provided the doorway and window functions are flat in the spectral region considered and the line widths of the sidebands are identical, the ratio of the fundamental $\omega_{\mathrm{G} 0}$, first $\omega_{\mathrm{G} 0} \pm \omega_{\mathrm{RBM}}$, and the second $\omega_{\mathrm{G} 0} \pm 2 \omega_{\mathrm{RBM}}$ sidebands is proportional to the $1: \gamma_{1}: \gamma_{2}$ ratio. As shown above, $\gamma_{1}$ and $\gamma_{2}$ are proportional to the anharmonic coupling constants, and therefore, the sideband intensities carry direct information on the mode coupling constants.

\section{QUANTUM CHEMICAL CALCULATIONS}

We used the Austin Model 1 (AM1) hamiltonian ${ }^{28}$ and the ESMD computational package ${ }^{13}$ to follow photoexcited adiabatic dynamics on ps timescales for all calculations presented in this letter. The ESMD approach calculates the excited-state potential energy as $E_{e}(\mathbf{q})=E_{g}(\mathbf{q})+\Omega(\mathbf{q})$ in the space of all nuclear coordinates $\mathbf{q}$ which span the entire $3 N-6$-dimensional space, $N$ being the total number of atoms in the molecule. Here $\Omega(\mathbf{q})$ is an electronic transition frequency to the lowest optically active excited state of the molecule calculated using the time-dependent Hartree-Fock approximation. The program uses efficient analytic derivatives of $E_{e}(\mathbf{q})$ with respect to each nuclear coordinate $q_{i}$ to calculate forces and subsequently to step along the excited-state hypersurface using these gradients. A standard Verlet molecular dynamics algorithm has been used for propagation of the Newtonian equations of motion along all $(i=1, \ldots, 3 N-6)$ nuclear degrees of freedom $q_{i}$. If no dissipative processes are included, the total molecular energy $E_{e}(\mathbf{q})$ is conserved. Subsequent analysis of the photoexcited trajectory in Fourier space allows us to identify periods of participating vibrational motions. Inclusion of an artificial dissipative force into the ESMD equations of motion allows us to determine the minimum of the excited-state potential energy surface $E_{e}(\mathbf{q})$ corresponding to the relaxed geometry.

Received 30 January 2006; accepted 1 June 2006; published 9 July 2006.

References

1. Dresselhaus, M. S., Dresselhaus, G. \& Avouris, P. (eds) Carbon Nanotubes : Synthesis, Structure, Properties and Applications (Springer, Berlin, 2001).

2. Treacy, M. M. J., Ebbesen, T. W. \& Gibson, J. M. Exceptionally high Young's modulus observed for individual carbon nanotubes. Nature 381, 678-680 (1996).

3. Tans, S. J., Verschueren, A. R. M. \& Dekker, C. Room-temperature transistor based on a single carbon nanotube. Nature 393, 49-52 (1998). 
4. Zhao, H. \& Mazumdar, S. Electron-electron interaction effects on the optical excitations of semiconducting single-walled carbon nanotubes. Phys. Rev. Lett. 93, 157402 (2004).

5. Korovyanko, O. J., Sheng, C.-X., Vardeny, Z. V., Dalton, A. B. \& Baughman, R. H. Ultrafast spectroscopy of excitons in single-walled carbon nanotubes. Phys. Rev. Lett. 92, 017403 (2004).

6. Wang, F., Dukovic, G., Brus, L. E. \& Heinz, T. F. The optical resonances in carbon nanotubes arise from excitons. Science 308, 838-841 (2005).

7. Spataru, C. D., Ismail-Beigi, S., Benedict, L. X. \& Louie, S. G. Excitonic effects and optical spectra of single-walled carbon nanotubes. Phys. Rev. Lett. 92, 077402 (2004).

8. Chang, E., Bussi, G., Ruini, A. \& Molinari, E. Exciton in carbon nanotubes: An Ab initio symmetry-based approach. Phys. Rev. Lett. 92, 196401 (2004).

9. LeRoy, B. J., Lemay, S. G., Kong, J. \& Dekker, C. Electrical generation and absorption of phonons in carbon nanotubes. Nature 432, 371-374 (2004).

10. Htoon, H., O'Connel, M. J., Doorn, S. K. \& Klimov, V. I. Single carbon nanotubes probed by photoluminescence excitation spectroscopy: the role of phonon assisted transitions. Phys. Rev. Let. 94, 127403 (2005).

11. Perebeinos, V., Tersoff, J. \& Avouris, P. Effect of exciton-phonon coupling in the calculated optical absorption of carbon nanotubes. Phys. Rev. Lett. 94, 027402 (2005).

12. Zavelani-Rossi, M. et al. Pulse compression over $170-\mathrm{THz}$ bandwidth in the visible by use of only chirped mirrors. Opt. Lett. 26, 1155-1157 (2001).

13. Tretiak, S., Saxena, A., Martin, R. L. \& Bishop, A. R. Conformational dynamics of photoexcited conjugated molecules. Phys. Rev. Lett. 89, 097402 (2002).

14. Rao, A. M. et al. Diameter-selective Raman scattering from vibrational modes in carbon nanotubes. Science 275, 187-191 (1997)

15. Pollard, W. T., Lee, S.-Y. \& Mathies, R. A. Wave packet theory of dynamic absorption spectra in femtosecond pump-probe experiments. J. Chem. Phys. 92, 4012-4029 (1990).

16. Kobayashi, T., Saito, T. \& Ohtani, H. Real-time spectroscopy of transition states in bacteriorhodopsin during retinal isomerization. Nature 414, 531-534 (2001).

17. Lanzani, G., Cerullo, G., Brabec, C. \& Sariciftci, N. S. Time domain investigation of the intrachain vibrational dynamics of a prototypical light-emitting conjugated polymer. Phys. Rev. Lett. 90, 047402 (2003)

18. Manzoni, C. et al. Intersubband exciton relaxation dynamics in single-walled carbon nanotubes. Phys. Rev. Lett. 94, 207401 (2005).

19. Lauret, J.-S. et al. Ultrafast carrier dynamics in single-wall carbon nanotubes. Phys. Rev. Lett. 90 057404 (2003).

20. Ostojic, G. N. et al. Interband recombination dynamics in resonantly excited single-walled carbon nanotubes. Phys. Rev. Lett. 92, 117402 (2004).
21. Ma, Y.-Z. et al. Ultrafast carrier dynamics in single-walled carbon nanotubes probed by femtosecond spectroscopy. J. Chem. Phys. 120, 3368-3373 (2004).

22. Zeiger, H. J. et al. Theory for displacive excitation of coherent phonons. Phys. Rev. B 45, 768-778 (1992).

23. Fantini, C. et al. Optical transition energies for carbon nanotubes from resonant raman spectroscopy: environment and temperature effects. Phys. Rev. Lett. 93, 147406 (2004).

24. Papadakis, S. J. et al. Resonant oscillators with carbon-nanotube torsion spring. Phys. Rev. Lett. 93 146101 (2004).

25. Dumitrică, T., Garcia, M. E., Jeschke, H. O. \& Yakobson, B. I. Selective cap opening in carbon nanotubes driven by laser-induced coherent phonons. Phys. Rev. Lett. 92, 117401 (2004).

26. Vrakking, M. J. J., Villeneuve, D. M. \& Stolow, A. Observation of fractional revivals of a molecular wave packet. Phys. Rev. A 54, R37-R40 (1996).

27. Brar, V. W. et al. Second-order harmonic and combination modes in graphite, single-wall carbon nanotube bundles, and isolated single-wall carbon nanotubes. Phys. Rev. B 66, 155418 (2002).

28. Dewar, M. J. S., Zoebisch, E. G., Healy, E. F. \& Stewart, J. J. P. AM1: A new general purpose quantum mechanical molecular model. J. Am. Chem. Soc. 107, 3902-3909 (1985).

29. Della Negra, F., Meneghetti, M. \& Menna, E. Microwave-assisted synthesis of a soluble single wall carbon nanotube derivative. Fuller. Nanotub. Carb. Nanostr. 11, 25-34 (2003).

30. Menna, E., Della Negra, F., Dalla Fontana, M. \& Meneghetti, M. Selectivity of chemical oxidation attack of single-wall carbon nanotubes in solution. Phys. Rev. B 68, 193412 (2003).

31. Mukamel, S. Principles of Nonlinear Optical Spectroscopy (Oxford Univ. Press, New York, 1995).

\section{Acknowledgements}

We thank G. Marcolongo for technical help and Z. V. Vardeny for useful discussions. M.M., G.L. and E.M. acknowledge financial support from MIUR (contracts PRIN-2004035502, FIRB-RBNE 033KMA, FIRB-RBNE01P4JF). The research at LANL is supported by the Center for Integrated Nanotechnology (CINT), Los Alamos LDRD Funds and the Office of Basic Energy Sciences, US Department of Energy. This support is gratefully acknowledged.

Correspondence and requests for materials should be addressed to G.L.

\section{Competing financial interests}

The authors declare that they have no competing financial interests.

Reprints and permission information is available online at http://npg.nature.com/reprintsandpermissions/ 